Pacific Journal of Mathematics

ON THE ENGEL MARGIN

Vol. 50, No. 1

September 1974 


\title{
ON THE ENGEL MARGIN
}

\author{
T. K. Teague
}

The marginal subgroup for any outer commutator word has been characterized by R. F. Turner-Smith. This paper considers the marginal subgroup $E(G)$ of $G$ for the Engel word $e_{2}(x, y)=[x, y, y]$ of length two. The principal result is that an element $a$ of $G$ is in $E(G)$ if and only if $[x, y, a][a, y, x]$ is a law in $G$. The method of proof relies upon properties of Engel elements established by W. Kappe.

Among other results are the following: (a) $E(G) / Z_{2}(G)$ is an elementary Abelian 3-group of central automorphisms on the commutator subgroup $G^{\prime}$. (b) If $Z(G) \cap \gamma_{3}(G)$ has no elements of order 3 or if $G^{\prime}$ is Cernikov complete, then $E(G)=$ $Z_{2}(G)$. (c) If $[G: E(G)]=m$ is finite, then the verbal subgroup $e_{2}(G)$ is finite with order dividing a power of $m$.

1. Notation and assumed results. Let $\phi\left(x_{1}, \cdots, x_{n}\right)$ be any word in the variables $x_{1}, \cdots, x_{n}$. The verbal subgroup $\phi(G)$ is the subgroup of $G$ generated by all elements of the form $\phi\left(a_{1}, \cdots, a_{n}\right)$ with $a_{1}, \cdots, a_{n}$ in $G$. We say $\phi$ is a law in $G$, or that $G$ is in the variety determined by $\phi$, if $\phi(G)=1$.

The associated marginal subgroup $\phi^{*}(G)$ of $G$ consists of all $a$ in $G$ such that $\phi\left(g_{1}, \cdots, a g_{i}, \cdots, g_{n}\right)=\phi\left(g_{1}, \cdots, g_{i}, \cdots, g_{n}\right)$ for every $g_{i}$ in $G, 1 \leqq i \leqq n$. We also refer to $\phi^{*}(G)$ as the $\phi$-margin of $G$.

For $x, y, a_{i}$ in $G$, define $[x, y]=x^{-1} y^{-1} x y=x^{-1} x^{y},\left[a_{1}, \cdots, a_{n}\right]=$ $\left[\left[a_{1}, \cdots, a_{n-1}\right], a_{n}\right]$, and $[x,(n+1) y]=[[x, n y], y]$. Similarly, for subgroups $H$ and $K$ of $G,[H, K]$ denotes the subgroup generated by all elements of the form $[h, k]$, where $h \in H, k \in K$. We define $[H,(n+$ 1) $K]=[[H, n K], K]$. If $H_{1}, \cdots, H_{n}$ are subgroups, then $\left[H_{1}, \cdots, H_{n}\right]=$ [[ $\left.\left.H_{1}, \cdots, H_{n-1}\right], H_{n}\right]$.

The word $\gamma_{1}=d_{0}=x$ is an outer commutator word of weight one. If $\theta=\theta\left(x_{1}, \cdots, x_{m}\right), \lambda=\lambda\left(y_{1}, \cdots, y_{n}\right)$ are outer commutator words of weights $m$ and $n$ respectively, then $\phi=\phi\left(x_{1}, \cdots, x_{m+n}\right)=$ $\left[\theta\left(x_{1}, \cdots, x_{m}\right), \lambda\left(x_{m+1}, \cdots, x_{m+n}\right)\right]$ is an outer commutator word of weight $m+n$. We write $\phi=[\theta, \lambda]$. Particular examples are the derived (or solvable) words, defined by $d_{n}=\left[d_{n-1}, d_{n-1}\right]$, and the nilpotent (or lower central) words, defined by $\gamma_{n+1}=\left[\gamma_{n}, \gamma_{1}\right]$.

The following two theorems appear in [15]:

THEOREM 1.1. For any group $G$ and word $\phi$,

(a) $\phi(G)$ is fully invariant in $G$ and $\phi^{*}(G)$ is characteristic in $G$.

(b) $\phi\left(\phi^{*}(G)\right)=1$. 
( c) if $K / \phi^{*}(G)$ is the center of $G / \phi^{*}(G)$, then $[K, \phi(G)]=1$. In particular, $\left[\phi^{*}(G), \phi(G)\right]=1$.

(d) if $H$ is a subgroup such that $G=H \phi^{*}(G)$, then $\phi^{*}(H)=$ $H \cap \phi^{*}(G)$ and $\phi(G)=\phi(H)$.

THEOREM 1.2. Let $\theta$ and $\lambda$ be two words in independent variables and $\phi=[\theta, \lambda]$. Then, in any group $G$,

(a) $\phi(G)=[\theta(G), \lambda(G)]$.

(b) if $U=C_{G}(\theta(G)), V=C_{G}(\lambda(G)), L / U=\lambda^{*}(G / U)$, and $M / V=$ $\theta^{*}(G / V)$, then $\phi^{*}(G)=L \cap M$.

An immediate result of Theorem $1.2(\mathrm{~b})$ is that $\gamma_{n+1}^{*}(G)=Z_{n}(G)$, the $n$th center of $G$. It is this theorem which makes possible a classification of marginal subgroups for all outer commutator words, since the variables in $\theta$ and $\lambda$ are independent of each other (see [16, p. 328]).

An element $x$ of $G$ is called a left (right) Engel element of $G$ if for every $y$ in $G$ there is a positive integer $n$ such that $[y, n x]=1$ $([x, n y]=1)$. The Engel word of length $n$ is $e_{n}(x, y)=[x, n y]$. We note that Theorem 1.2(b) can not be used to determine $e_{n}^{*}(G)$, since $e_{n-1}(x, y)$ and $y$ are not independent.

For $H$ a subgroup of $G,[G: H]$ is the index of $H$ in $G$. If $H$ is a proper (normal) subgroup of $G$, write $H<G(H \triangleleft G)$. If $G$ is isomorphic to a subgroup of a group $K$, write $G \cong K . C_{G}(H)$ is the centralizer of $H$ in $G$. For $x$ in $G, x^{G}$ denotes the subgroup generated by all conjugates of $x$ in $G$.

2. The Engel margin. In this section "Engel word" will mean "Engel word of length two". We write $M(G)=d_{2}^{*}(G)$ and $E(G)=$ $e_{2}^{*}(G)$ for the metabelian and Engel margins of $G$ respectively.

Recall that $\left[Z_{n}(G), \gamma_{m}(G)\right] \subseteq Z_{n-m}(G)$ for all positive integers $m$ and $n$.

LEMMA 2.1. In any group $G$,

( a ) $d_{n}^{*}(G) / C_{G}\left(d_{n-1}(G)\right)=d_{n-1}^{*}\left(G / C_{G}\left(d_{n-1}(G)\right)\right)$. In particular, $M(G)=$ $\{a \in G \mid[[a, x],[y, z]]$ is a law in $G\}$.

(b) $Z_{n(n+1) / 2}(G) \subseteq d_{n}^{*}(G)$. In particular, $Z_{3}(G) \subseteq M(G)$.

Proof. Part (a) follows from Theorem 1.2(b) with $\theta=\lambda=d_{n-1}$.

We prove (b) by induction on $n$. For $n=1, Z_{1}(G) \subseteq d_{1}^{*}(G)=Z(G)$. For $n>1$, let $\bar{G}=G / C_{G}\left(d_{n-1}(G)\right)$. Then

$$
\overline{d_{n}^{*}(G)}=d_{n-1}^{*}(\bar{G}) \supseteq Z_{n(n-1) / 2}(\bar{G})
$$

by part (a) and the induction hypothesis. Furthermore, 


$$
\left[Z_{n(n+1) / 2}(G), n(n-1) / 2(G)\right] \subseteq Z_{n(n+1) / 2-n(n-1) / 2}(G)=Z_{n}(G)
$$

and $\left[Z_{n}(G), d_{n-1}(G)\right] \subseteq\left[Z_{n}(G), \gamma_{n}(G)\right]=1$ so that

$$
\left[Z_{n(n+1) / 2}(G), n(n-1) / 2(G)\right] \subseteq C_{G}\left(d_{n-1}(G)\right) .
$$

Consequently,

$$
\overline{Z_{n(n+1) / 2}(G)} \subseteq Z_{n(n-1) / 2}(\bar{G}) \subseteq \overline{d_{n}^{*}(G)}
$$

and $Z_{n(n+1) / 2}(G) \subseteq d_{n}^{*}(G) C_{G}\left(d_{n-1}(G)\right)=d_{n}^{*}(G)$, as desired.

We define $E_{1}(G)=\{a \in G \mid[a x, y, y]=[x, y, y]$ for all $x, y \in G\}$ and $L(G)=\{a \in G \mid[a, x, x]$ is a law in $G\}$ to be the subgroup of right Engel elements of length two. It is not difficult to show that $E(G) \subseteq E_{1}(G)$ and $E_{1}(G)$ is a characteristic subgroup of $G$.

The following properties of $L(G)$ were established by W. Kappe in [6]:

LemmA 2.2. In any group $G$, where $a \in L(G), g, h, \in G$,

(a) $L(G)$ is a characteristic subgroup of $G$.

(b) $[a, g, h]=[a, h, g]^{-1}$.

(c) $[a,[g, h]]=[a, g, h]^{2}$.

(d) $[a, g,[h, g]]=1$.

(e ) $a^{4} \in Z_{3}(G)$.

THEOREM 2.3. In any group $G$,

(a) $Z_{2}(G) \subseteq E(G) \subseteq L(G)$.

(b) $E_{1}(G)=\left\{a \in G \mid[a, x] \in C_{G}\left(x^{G}\right)\right.$ for all $\left.x \in G\right\}=L(G)$.

(c) $[a, x] \in C_{G}\left(x^{G}\right) \cap C_{G}(a)$ for all $a \in E_{1}(G), x \in G$. Furthermore, $[a, x]^{r s}=\left[a^{r}, x^{s}\right]$ for all integers $r$ and $s$.

(d) $a^{G}$ and $x^{L(G)}$ are Abelian for all $a$ in $L(G), x$ in $G$.

(e) $E_{1}(G) \subseteq C_{G}\left(\left(x^{G}\right)^{\prime}\right) \triangleleft G$ for all $x$ in $G$.

Proof. Part (a) follows immediately from the definitions.

(b) Let $a \in E_{1}(G)$. Then $[a y, x, x]=[y, x, x]$ for all $x, y$ in $G$. This is equivalent to saying that $1=\left[[a y, x][y, x]^{-1}, x\right]=\left[[a, x]^{y} \times\right.$ $\left.[y, x][y, x]^{-1}, x\right]=\left[[a, x]^{y}, x\right]$ for all $x, y$ in $G$. Since $x$ and $y$ are independent, we may conclude that $a$ is in $E_{1}(G)$ if and only if $1=$ $\left[a, x, x^{y}\right]$ for all $x, y$ in $G$ or, equivalently, $[a, x] \in C_{G}\left(x^{G}\right)$ for all $x$.

That $E_{1}(G) \subseteq L(G)$ follows from $\left[a, x, x^{y}\right]=1$ by letting $y=1$. Finally, let $a \in L(G)$. We have for $x, y$ in $G$ that

$$
\left[a, x, x^{y}\right]=[a, x, x[x, y]]=[a, x,[x, y]][a, x, x]^{[x, y]} .
$$

From the definition of $L(G)$ we must have that $[a, x, x]=1$. By Lemma 2.2(d) we also have that $[a, x,[x, y]]=1$. Hence $\left[a, x, x^{y}\right]=1$ and $a \in E_{1}(G)$. 
(c) Since $a$ is a right Engel element, we have that $[a, x]$ is in $C_{G}(a)$ by [6, Lemma 2.1]. Part (b) says that $[a, x] \in C_{G}\left(x^{G}\right)$ for all $x$ in $G$. The remainder of part (c) follows from [13, Theorem 3.4.4].

(d) From part (c) we see that $a^{x}=a[a, x] \in C_{G}(a)$, since $a$ and $[a, x]$ are in $C_{G}(a)$. This implies that $a^{G}$ is Abelian.

The proof that $x^{L(G)}$ is Abelian follows similarly from the observation that $x^{a}=x[x, a],[x, a] \in C_{G}\left(x^{G}\right) \subseteq C_{G}(x)$.

(e) By part (c) we may conclude that $\left[a, x^{y}\right] \in C_{G}\left(\left(x^{y}\right)^{G}\right)=C_{G}\left(x^{G}\right)$ for all $a$ in $E_{1}(G), x, y$ in $G$.

Let $a \in E_{1}(G)$. By Lemma $2.2(\mathrm{c})$, we have $\left[a,\left[x^{w}, x^{z}\right]\right]=$ $\left[\left[a, x^{w}\right], x^{z}\right]^{2}=1$. This implies that $a \in C_{G}\left(\left(x^{G}\right)^{\prime}\right)$.

Theorem 2.4. In any group $G, E(G)=\{a \in G \mid[x, a, y][x, y, a]=1$ for all $x, y$ in $G\}$.

Proof. Set $E_{2}(G)=\{a \in G \mid[x, a y, a y]=[x, y, y]$ for all $x, y$ in $G\}$. We see then that $E(G)=E_{1}(G) \cap E_{2}(G)$. Let $S$ be the set described on the right in the statement of the theorem. Suppose $a \in S, x \in G$. Then $1=[x, a, x][x, x, a]=[x, a, x]$. This implies that $a \in E_{1}(G)=L(G)$. Since also $E(G) \subseteq E_{1}(G)$, it suffices to show that $E(G) \cap E_{1}(G)=E_{1}(G) \cap$ $E_{2}(G)=E_{1}(G) \cap S$. Then, for $x, y$ in $G, a \in E_{1}(G) \cap E_{2}(G)$ if and only if

$$
\begin{aligned}
{[x, y, y] } & =[x, a y, a y] \\
& =[x, a y, y][x, a y, a]^{y} \\
& =\left[[x, y][x, a]^{y}, y\right]\left[[x, y][x, a]^{y}, a\right]^{y} \\
& =[x, y, y]^{[x, a]^{y}}\left[[x, a]^{y}, y\right][x, y, a]^{[x, a]^{y} y}\left[[x, a]^{y}, a\right]^{y} .
\end{aligned}
$$

By assumption, $[a, x] \in C_{G}\left(x^{G}\right)$. Since $C_{G}\left(x^{G}\right) \triangleleft G$, we also have that $[a, x]^{y} \in C_{G}\left(x^{a}\right)$. Consequently, conjugation by $[x, a]^{y}$ is irrelevant in the last statement above because all the commutators are in $x^{G}$. Therefore, the above is equivalent to

$$
[x, y, y]=[x, y, y]\left[[x, a]^{y}, y\right][x, y, a]^{y}\left[[x, a]^{y}, a\right]^{y}
$$

or

$$
1=[x, a, y][x, y, a]\left[[x, a]^{y}, a\right]
$$

for all $x, y \in G, a \in E(G)$.

Now $a$ and $[x, a]^{y}$ are elements of $a^{G}$. By Theorem 2.3(d), $a^{G}$ is Abelian. This implies that $\left[[x, a]^{y}, a\right]=1$. Therefore, $E(G)$ is contained in the set $S$.

We have already shown that $S$ is a subset of $E_{1}(G)=L(G)$. Consequently, all the above arguments are reversible and we may conclude that $S=E(G)$. 
Lemma 2.5. (a) $E(G) \cap C_{G}\left(G^{\prime}\right)=Z_{2}(G)$.

(b) $[x, a, y]=[a, y, x]$ for all $x, y$ in $G, a$ in $L(G)$.

Proof. (a) We need only verify that $E(G) \cap C_{G}\left(G^{\prime}\right) \subseteq Z_{2}(G)$ by Theorem 2.3(a) and the remark before Lemma 2.1. Let $a \in E(G) \cap$ $C_{G}\left(G^{\prime}\right)$. By Theorem 2.4, $1=[x, a, y][x, y, a]$ for all $x, y$ in $G$. But $a \in C_{G}\left(G^{\prime}\right)$ implies that $[x, y, a]=1$ and thus that $[x, a, y]=1$ for all $x, y$ in $G$. Hence $a \in Z_{2}(G)$.

(b) $[a, y, x]=[a, x, y]^{-1}$ by Lemma 2.2(b), $=\left[[x, a]^{-1}, y\right]^{-1}=$ $\left(\left([x, a, y]^{-1}\right)^{-1}\right)^{[a, x]}=[x, a, y]$ since $[a, x] \in C_{G}\left(x^{G}\right)$ by Theorem 2.3(c).

From Theorem 2.4 and Lemma 2.5(b) we have our characterization of $E(G)$ :

TheOREM 2.6. For any group $G, E(G)=\{a \in G \mid[x, y, a][a, y, x]$ is a law in $G$ \}.

Corollary 2.7. For any $a \in E(G),[a, G, G]^{3}=\left[\alpha^{3}, G, G\right]=1$.

Proof. Let $x, y \in G$. By Theorem 2.6, $[x, y, a][a, y, x]=1$. Then $[x, y, a]=[a,[x, y]]^{-1}=\left([a, x, y]^{2}\right)^{-1}$ by Lemma 2.2(c), $=[a, y, x]^{2}$ by Lemma 2.2(b). Hence $1=[x, y, a][a, y, x]=[a, y, x]^{2}[a, y, x]=[a, y, x]^{3}$.

By Theorem 2.3(d) we have that $a^{G}$ is Abelian. Hence $[a, x, y]^{3}=1$ for all $x, y \in G$ implies $[a, G, G]$ has exponent dividing three, and $[a, x, y]^{3}=\left[a^{3}, x, y\right]=1$.

CoRollary 2.8. For any group $G, E(G) \subseteq Z_{3}(G) \subseteq M(G)$.

Proof. Let $a \in E(G)$. By Lemma 2.2(e) we have that $a^{4} \in Z_{3}(G)$. Since also $a^{3} \in Z_{2}(G) \subseteq Z_{3}(G)$ by Corollary 2.7, it follows that $a \in Z_{3}(G)$.

We recall a theorem of $\mathrm{F}$. W. Levi (see [12]): If $e_{2}$ is a law in a group $G$, then $G$ is nilpotent of class at most three and $\gamma_{3}(G)$ has exponent dividing three. This, together with Theorem 1.1(b), yields the first statement in the following:

THEOREM 2.9. $E(G)$ is nilpotent of class no greater then three and metabelian, and $\gamma_{3}(E(G))$ has exponent dividing three. If $C_{G}\left(G^{\prime}\right) \subseteq$ $E(G)$, then $M(G)=Z_{3}(G)$.

Proof. Suppose $C_{G}\left(G^{\prime}\right) \subseteq E(G)$. By Lemma 2.5(a) this implies that $C_{G}\left(G^{\prime}\right)=Z_{2}(G)$. From Lemma 2.1(a), $M(G) / C_{G}\left(G^{\prime}\right)=Z\left(G / C_{G}\left(G^{\prime}\right)\right)$. Hence $M(G)=Z_{3}(G)$. 
THEOREM 2.10. Let $G$ be a group, $M=M(G), E_{1}=E_{1}(G)=L(G)$. Then

(a) $\left[G^{\prime}, M, E_{1}\right]=\left[G^{\prime}, E_{1}, M\right]=\left[M, G, G^{\prime}\right]=1$.

(b) $\left[G, M^{\prime}, E_{1}\right]=\left[M^{\prime}, E_{1}, G\right]=\left[G^{\prime}, M^{\prime}\right]=1$. In particular, $\left[M^{\prime}, E_{1}\right] \subseteq$ $Z(G)$.

Proof. (a ) By Lemma 2.1(a), $[M, G] \subseteq C_{G}\left(G^{\prime}\right) \cap G^{\prime}=Z\left(G^{\prime}\right)$ so that $1=\left[M, G, G^{\prime}\right]$. Now let $a \in E_{1}, m \in M, x \in G^{\prime}$. By Lemma 2.2(c), $[a,[m, x]]=[a, m, x]^{2}=1$. This implies $\left[G^{\prime}, M, E_{1}\right]=1$. Consequently $\left[G^{\prime}, E_{1}, M\right]=1$ by [13, Theorem 3.4.8(i)].

(b) As in the proof of part (a), we have $M^{\prime} \subseteq Z\left(G^{\prime}\right)$ so that $1=\left[G^{\prime}, M^{\prime}\right]$. Let $a \in E_{1}, x \in M^{\prime}, g \in G$. Then $[a,[g, x]]=[a, g, x]^{2}=1$. Hence $\left[M^{\prime}, G, E_{1}\right]=1$ and, as above, $\left[M^{\prime}, E_{1}, G\right]=1$.

3. Central automorphisms on $G^{\prime}$. It follows from Theorem 2.10(a) that $\left[M(G), G^{\prime}\right] \subseteq Z\left(G^{\prime}\right)$. This implies that $M(G) / C_{G}\left(G^{\prime}\right)$ acts as an Abelian group of central automorphisms on $G^{\prime}$. Then

$$
\left(E_{1}(G) \cap M(G)\right) /\left(E_{1}(G) \cap C_{G}\left(G^{\prime}\right)\right) \sqsubseteq M(G) / C_{G}\left(G^{\prime}\right)
$$

is also such a group. Denote the corresponding group of automorphisms on $G^{\prime}$ by $\mathfrak{2}_{2}$. Furthermore,

$$
E(G) / Z_{2}(G)=(E(G) \cap M(G)) /\left(E(G) \cap C_{G}\left(G^{\prime}\right)\right) \sqsubseteq \mathfrak{U}_{2}
$$

by Lemma 2.5(a) and Corollary 2.8. Let $\mathfrak{A}_{1} \subseteq \mathfrak{U}_{2}$ denote the corresponding group of automorphisms. From Corollary 2.7 we see that $E(G) / Z_{2}(G)$ has exponent 3. Hence $\mathfrak{H}_{1}$ is an elementary Abelian 3group of central automorphisms on $G^{\prime}$.

Theorem 3.1. (a) If the exponent $\operatorname{Exp}\left(Z\left(G^{\prime}\right)\right)=n$ is finite, then $\operatorname{Exp}\left(\mathfrak{H}_{2}\right)$ divides $n$.

(b) If $G^{\prime}$ is a p-group, $\mathfrak{A} \subseteq \mathfrak{A}_{2}$ is periodic, then $\mathfrak{X}$ is a p-group.

(c) Assume $G^{\prime}$ is polycyclic; that is, $G^{\prime}$ has a finite ascending normal series with cyclic factors. Then $E(G) / Z_{2}(G)$ is finite.

Proof. (a) Suppose $Z\left(G^{\prime}\right)$ has exponent $n$. Then, for $x \in G^{\prime}$, $a \in \mathfrak{N}_{2}, 1=[x, a]^{n}=\left[x, a^{n}\right]$ by Theorem 2.3(c). Consequently, $a^{n}=1$ and $\mathfrak{U}_{2}$ has exponent dividing $n$.

(b) Now assume $\mathfrak{X}$ is periodic. By Theorem 2.10(a) we may conclude that $\left[G^{\prime}, M(G), E_{1}(G)\right]=\left[G^{\prime}, \mathfrak{N}, \mathfrak{X}\right]=1$. Thus $\mathfrak{X}$ stabilizes the normal series $1 \triangleleft\left[G^{\prime}, \mathfrak{N}\right] \triangleleft G^{\prime}$ of $G^{\prime}$. By [1, Corollary 5.3.3] we have that $\mathfrak{A}$ is a $p$-group.

(c) Smirnov [14] has shown that a solvable group of automorphisms of a polycyclic group is polycyclic. Since then $\mathfrak{U}_{1}$ is finitely generated, it must be finite. 
THEOREM 3.2. If $\mathfrak{A}_{2} \neq 1$ is not torsionfree, then $G^{\prime}$ has a proper subgroup of finite index and $Z\left(G^{\prime}\right)$ is not torsionfree.

Proof. For $1 \neq \alpha \in \mathfrak{A}_{2}$, the homomorphism from $G^{\prime}$ into $Z\left(G^{\prime}\right)$ defined by $f_{\alpha}(x)=[x, \alpha]$ for each $x$ in $G^{\prime}$ is nontrivial. We choose $a \in E_{1}(G) \cap M(G) \backslash E_{1}(G) \cap C_{G}\left(G^{\prime}\right)$ such that $[x, \alpha]=[x, \alpha]$ for all $x$ in $G^{\prime}$. If $\alpha$ has finite order, then there is an integer $n$ such that $a^{n} \in C_{G}\left(G^{\prime}\right)$. Thus $1=[x, \alpha]^{n}=\left[x, a^{n}\right]$ and $G^{\prime} / \operatorname{Ker} f_{\alpha} \subseteq Z\left(G^{\prime}\right)$ is a nontrivial direct sum of cyclic groups each of order bounded by $n$. In particular, there are subgroups $H$ and $C$ of $G^{\prime}$ such that $G^{\prime} / \operatorname{Ker} f_{\alpha}=H / \operatorname{Ker} f_{\alpha}+$ $C / \operatorname{Ker} f_{\alpha}$ and $C / \operatorname{Ker} f_{\alpha}$ is nontrivial and finite. Consequently $H<G^{\prime}$ and $G^{\prime} / H \cong C / \operatorname{Ker} f_{\alpha}$ is finite.

Let $1 \neq \alpha \in \mathfrak{A}_{2}, o(\alpha)=n<\infty$. Then there is an $x \in G^{\prime}$ such that $1 \neq[x, \alpha] \in Z\left(G^{\prime}\right)$. But $[x, \alpha]^{n}=\left[x, \alpha^{n}\right]=1$ so that the order of $[x, \alpha]$ divides $n$.

CoROllaRY 3.3. If $E(G)>Z_{2}(G)$, then $G^{\prime}$ has a proper subgroup of finite index.

Proof. If $E(G)>Z_{2}(G)$, then $\mathfrak{X}_{1}$ is a nontrivial torsion subgroup of $\mathfrak{N}_{2}$. Hence $\mathfrak{A}_{2} \neq 1$ is not torsionfree and the theorem applies.

It is known that no complete, or even Černikov complete, group can have a proper subgroup of finite index (see [7, p. 234]). From this fact we derive part of the following:

COROLLARY 3.4. If $G^{\prime}$ is Černikov complete, or if $Z(G) \cap \gamma_{3}(G)$ has no elements of order three, then $E(G)=Z_{2}(G)$.

Proof. We shall show that $\mathfrak{N}_{1}=1$. By Corollary 2.8, $E(G) \subseteq$ $Z_{3}(G)$. Hence $\left[G^{\prime}, E(G)\right]=\left[G^{\prime}, \mathfrak{N}_{1}\right] \subseteq Z(G) \cap \gamma_{3}(G)$.

Let $a \in \mathfrak{N}_{1}, x \in G^{\prime}$. Then, by Corollary 2.7 and Theorem 2.3(c), $1=\left[x, a^{3}\right]=[x, a]^{3}$. By hypothesis, this implies that $1=[x, a]$. Consequently $a=1$.

EXAMPLE 3.5. We now construct a group $G$ such that $Z_{2}(G)<$ $E(G)<Z_{3}(G)$.

Let $H=\left\langle a_{1}, a_{2}, a_{3}: x^{3}\right\rangle$. Levi and van der Waerden [8] have shown that $H$ has nilpotence class exactly three and is in the variety determined by $e_{2}$. Hence $E(H)=H=Z_{3}(H)>Z_{2}(H)$. Let $K$ be any group of nilpotence class at least three having no elements of order three (see for example [12, p. 198]). By Corollary 3.4, $E(K)=Z_{2}(K)<$ $Z_{3}(K) \subseteq K$. Letting $G=H \times K$, we see that $E(G)=E(H) \times E(K)=$ $H \times Z_{2}(K)$. Hence $Z_{2}(G)<E(G)<Z_{3}(G)$. 
Remark 3.6. Define $N_{A}(G)=\bigcap\left\{N_{G}(H) \mid H\right.$ maximal Abelian subgroup of $G\}$ to be the $A$-Norm of $G$. Kappe [6] has shown that $a \in N_{A}(G)$ if and only if $[g, h]=1$ for $g, h$ in $G$ implies that $[a, g, h]=1$. From Theorem 2.6 it follows immediately that $E(G) \subseteq N_{A}(G) \subseteq E_{1}(G)$.

4. Finiteness conditions. We shall say that a word $\phi$ satisfies the Schur-Baer property if $\left[G: \phi^{*}(G)\right]=m$ finite implies $\phi(G)$ finite with order which divides a power of $m$ for all groups $G$.

Schur showed that $\gamma_{2}$ satisfies the Schur-Baer property; Baer extended this result to any outer commutator word $\phi$ (see [15]).

Recall that a group $G$ is residually finite if for every $x$ in $G$, $x \neq 1$, there is a normal subgroup $N_{x}$ of $G$ such that $x \notin N_{x}$ and $G / N_{x}$ is finite. A group is locally residually finite if every finitely generated subgroup is residually finite.

We shall need the following theorem. For a proof (due to $\mathrm{P}$. Hall), see [15, Theorem 2].

THEOREM 4.1. If $\phi$ generates a locally residually finite variety, then $\phi$ satisfies the Schur-Baer property.

THEOREM 4.2. If $\dot{\phi} \in\left\{e_{2}, e_{3}\right\}$, then $\phi$ satisfies the Schur-Baer property.

Proof. Suppose $\phi=e_{2}$. A group in the variety generated by $\dot{\phi}$ is nilpotent by Levi's Theorem. A finitely generated nilpotent group is residually finite by $P$. Hall [4]. Therefore, a finitely generated group in the variety generated by $\phi$ is residually finite and Theorem 4.1 applies.

Let $\phi=e_{3}$. Heineken [5] has shown that a group in the variety generated by $\phi$ is locally nilpotent. Hence a finitely generated group in this variety is also residually finite and the theorem follows as above.

Recall that a group is an $S N^{*}$ group if it possesses an ascending normal series with Abelian factors (see [7]). Also, the unique maximum locally nilpotent normal subgroup of a group is called its HirschPlotkin radical (see [12]).

We note that in P. Hall's proof of Theorem 4.1 that we may extend the result somewhat if we put some restrictions on $G$ itself. That is, if $\phi^{*}(G)$ is locally residually finite for all $G$ in some quotientand subgroup-closed class $\Sigma$, then $\phi$ satisfies the Schur-Baer property for all $G$ in $\Sigma$.

THEOREM 4.3. If $G$ satisfies the maximum or the minimum condition, or if $G$ is an $S N^{*}$ group, then $e_{n}$ satisfies the Schur-Baer property for $G$. 
Proof. Suppose $G$ satisfies the maximum condition. Then, by [12, Theorem VI. 8. j], we have that the set of left Engel elements (of all lengths) is the Hirsch-Plotkin radical $R$. Since then $e_{n}^{*}(G) \subseteq R$ is locally nilpotent, it is locally residually finite. By the preceding remark, we have that $e_{n}$ satisfies the Schur-Baer property for $G$.

Vilyacer [18] has shown that an Engel group satisfying the minimum condition is locally nilpotent. Plotkin [11] has proved that an Engel group which is also an $S N^{*}$ group is locally nilpotent. Hence the remainder of the theorem follows as above.

The validity of the Schur-Baer property in general is one of several conjectures which have been proposed for the group functions $\phi$ and $\phi^{*}$ (see [9] and [16]). Modified solutions of two of these come from the following lemma.

LeMma 4.4. Suppose $G$ is in a class of groups in which the Schur-Baer property is satisfied locally for $\phi$. If $G$ is locally residually finite and $\phi$ is finite-valued on $G$, then $\phi(G)$ is finite.

Proof. This follows from the arguments used in the proofs of Proposition 1 and its two corollaries in [17].

We note in particular in these proofs that there is a finitely generated subgroup $H$ of $G$ such that $\phi(H)=\phi(G)$. It follows that $H / \phi^{*}(H)$ is finite. Since $H$ and $\phi$ satisfy the Schur-Baer property, $\dot{\phi}(H)=\phi(G)$ is finite.

The following two theorems are immediate from these observations.

THEOREM 4.5. If $\phi \in\left\{e_{2}, e_{3}\right\}, G$ is locally residually finite, and $\phi$ is finite-valued on $G$, then $\phi(G)$ is finite.

THEOREM 4.6. If $\phi \in\left\{e_{2}, e_{3}\right\}, \phi$ is finite-valued on $G$, and $G$ is finitely generated and residually finite, then $G / \phi^{*}(G)$ is finite.

\section{REFERENCES}

1. D. Gorenstein, Finite Groups, Harper and kow, New York, 1968.

2. P. Hall, Verbal and marginal subgroups, J. Reine Angew Math., 182 (1940), 156-157.

3. - Nilpotent groups, Report to the Canad. Math. Congress, 1957.

4. - On the finiteness of certain soluble groups, Proc. London Math. Soc., (3),

9 (1959), 595-622.

5. H. Heineken, Engelsche Elemente der Länge drei, Illinois J. Math., 5 (1961), 681707.

6. W. Kappe, Die A-Norm einer Gruppe, Illinois J. Math., 5 (1961), 187-197.

7. A. G. Kurosh, The Theory of Groups, Vol. 2, Chelsea, New York, 1960.

8. F. Levi and B. L. van der Waerden, Über eine besondere Klasse von Gruppen, Abhandl. Math. Sem. Univ. Hamburg, 9 (1932), 154-158. 
9. Ju. I. Merzljakov, Verbal and marginal subgroups of linear groups, Soviet Math. Dokl., 8 (1967), 1538-1541.

10. H. Neumann, Varieties of Groups, Ergebnisse der Mathematik und ihrer Grenzgebiete (N. S.), Band 37, Springer-Verlag, New York, 1967.

11. B. I. Plotkin, On some criteria of locally nilpotent groups, Uspehi Math. Nauk (N. S.), 9 (1954), no 3, 181-186. (Russian)

12. E. Schenkman, Group Theory, D. Van Nostrand, Princeton, N. J., 1965.

13. W. R. Scott, Group Theory, Prentice-Hall, Englewood Cliffs, N. J., 1964.

14. D. M. Smirnov, On groups of automorphisms of solvable groups, Mat. Sb., 32 (74) (1953), 365-384. (Russian)

15. P. W. Stroud, On a property of verbal and marginal subgroups, Proc. Cambridge Phil. Soc., 61 (1965), 41-48.

16. R. F. Turner-Smith, Marginal subgroup properties for outer commutator words, Proc. London Math. Soc. (3), 14 (1964), 321-341.

17. - Finiteness conditions for verbal subgroups, Journal London Math. Soc., 41 (1966), 166-176.

18. V. G. Vilyacer, On the theory of locally nilpotent groups, Uspehi Math. Nauk (N. S.), 13 (1958), no. 2, 284-285. (Russian)

Received August 29, 1972. This paper represents part of the author's Ph. D. dissertation written at Michigan State University under Professor Richard E. Phillips.

HENDRIX COLLEGE 


\section{PACIFIC JOURNAL OF MATHEMATICS}

\section{EDITORS}

RICHARD ARENS (Managing Editor) University of California

Los Angeles, California 90024

R. A. BeaUmont

University of Washington Seattle, Washington 98105
J. DUGUNDJI*

Department of Mathematics University of Southern California Los Angeles, California 90007

D. Gilbarg and J. Milgram Stanford University

Stanford, California 94305

\section{ASSOCIATE EDITORS}

E. F. BECKENBACH

B. H. NeUmanN

F. WOLF

K. YoSHIDA

\section{SUPPORTING INSTITUTIONS}

\section{UNIVERSITY OF BRITISH COLUMBIA CALIFORNIA INSTITUTE OF TECHNOLOGY UNIVERSITY OF CA.LIFORNIA MONTANA STATE UNIVERSITY UNIVERSITY OF NEVADA NEW MEXICO STATE UNIVERSITY OREGON STATE UNIVERSITY UNIVERSITY OF OREGON OSAKA UNIVERSITY}

UNIVERSITY OF SOUTHERN CALIFORNIA STANFORD UNIVERSITY UNIVERSITY OF TOKYO UNIVERSITY OF UTAH WASHINGTON STATE UNIVERSITY UNIVERSITY OF WASHINGTON AMERICAN MATHEMATICAL SOCIETY NAVAL WEAPONS CENTER

* C. R. DePrima California Institute of Technology, Pasadena, CA 91109, will replace J. Dugundji until August 1974. 


\section{Pacific Journal of Mathematics}

\section{Vol. 50, No. $1 \quad$ September, 1974}

Gail Atneosen, Sierpinski curves in finite 2-complexes.............. 1

Bruce Alan Barnes, Representations of $B^{*}$-algebras on Banach spaces .... 7

George Benke, On the hypergroup structure of central $\Lambda(p)$ sets ....... 19

Carlos R. Borges, Absolute extensor spaces: a correction and an

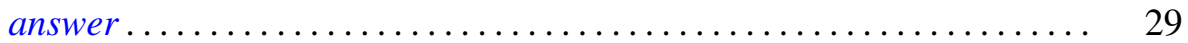

Tim G. Brook, Local limits and tripleability .................. 31

Philip Throop Church and James Timourian, Real analytic open maps .... 37

Timothy V. Fossum, The center of a simple algebra ............... 43

Richard Freiman, Homeomorphisms of long circles without periodic

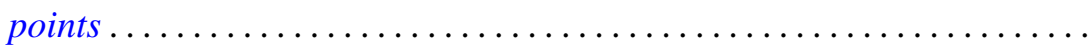

B. E. Fullbright, Intersectional properties of certain families of compact

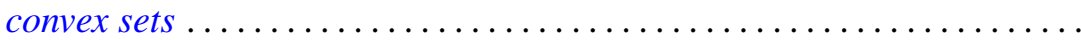

Harvey Charles Greenwald, Lipschitz spaces on the surface of the unit

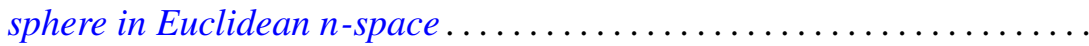

Herbert Paul Halpern, Open projections and Borel structures for

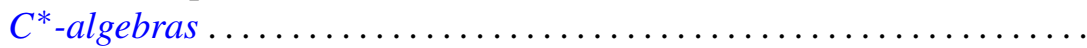

Frederic Timothy Howard, The numer of multinomial coefficients divisible

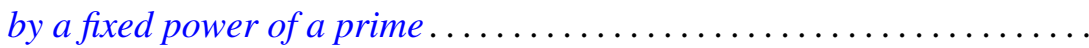

Lawrence Stanislaus Husch, Jr. and Ping-Fun Lam, Homeomorphisms of manifolds with zero-dimensional sets of nonwandering points........ 109

Joseph Edmund Kist, Two characterizations of commutative Baer rings ...

Lynn McLinden, An extension of Fenchel's duality theorem to saddle functions and dual minimax problems ................

Leo Sario and Cecilia Wang, Counterexamples in the biharmonic classification of Riemannian 2-manifolds...

Saharon Shelah, The Hanf number of omitting complete types ...

Richard Staum, The algebra of bounded continuous functions into a

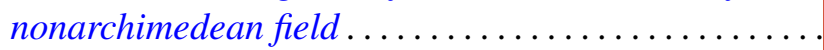

James DeWitt Stein, Some aspects of automatic continuity ..

Tommy Kay Teague, On the Engel margin

John Griggs Thompson, Nonsolvable finite groups all of whose local subgroups are solvable, $V \ldots \ldots \ldots \ldots \ldots \ldots \ldots \ldots$

Kung-Wei Yang, Isomorphisms of group extensions 